Original Article

\title{
Dry cupping for plantar fasciitis: a randomized controlled trial
}

\author{
Weiqing Ge, DPT, PhD ${ }^{1)^{*}}$, Chelsea Leson, DPT ${ }^{1)}$, Corey Vukovic, DPT ${ }^{1)}$
}

1) Department of Physical Therapy, Youngstown State University: One University Plaza, Youngstown, $\mathrm{OH} 44555$, USA

\begin{abstract}
Purpose] The purpose of this study was to determine the effects of dry cupping on pain and function of patients with plantar fasciitis. [Subjects and Methods] Twenty-nine subjects (age 15 to 59 years old, 20 females and 9 males), randomly assigned into the two groups (dry cupping therapy and electrical stimulation therapy groups), participated in this study. The research design was a randomized controlled trial (RCT). Treatments were provided to the subjects twice a week for 4 weeks. Outcome measurements included the Visual Analogue Pain Scale (VAS) (at rest, first in the morning, and with activities), the Foot and Ankle Ability Measure (FAAM), the Lower Extremity Functional Scale (LEFS), as well as the pressure pain threshold. [Results]The data indicated that both dry cupping therapy and electrical stimulation therapy could reduce pain and increase function significantly in the population tested, as all the $95 \%$ Confidence Intervals (CIs) did not include 0 except for the pressure pain threshold. There was no significant difference between the dry cupping therapy and electrical stimulation groups in all the outcome measurements. [Conclusion] These results support that both dry cupping therapy and electrical stimulation therapy could reduce pain and increase function in the population tested.

Key words: Pain, Function, Foot
\end{abstract}

(This article was submitted Dec. 10, 2016, and was accepted Feb. 13, 2017)

\section{INTRODUCTION}

Adult heel pain is usually caused by plantar fasciitis ${ }^{1-4)}$, the most common foot condition diagnosed and treated by healthcare providers ${ }^{5)}$. The onset of pain is usually gradual and typically occurs at the plantar medial heel ${ }^{6}$. . Most patients experience pain and tightness when taking their initial steps in the morning immediately after standing up from bed or after a period of prolonged rest such as sitting at the desk during the day ${ }^{1,3,5)}$. The pain usually improves after walking for a short period of time but could intensify after prolonged weight-bearing activities, including standing, walking, or running. Plantar fasciitis affects millions of Americans every or 10 percent of the population of the United States ${ }^{5,7)}$. Economic costs from third-party payers relating to the treatment of plantar fasciitis have been estimated to range from $\$ 192$ to $\$ 376$ million per year ${ }^{8)}$.

The plantar fascia is a thick fibrous aponeurosis formed from 3 bands of dense connective collagen fibers that attach proximally to the medial calcaneal tuberosity and fans distally to the flexor tendon sheaths and the base of the proximal phalanges $4,9,10$ ). This important structure provides the static and dynamic supports for the arch of the foot by transmitting forces between the heel and forefoot during weight-bearing activities. As the terminology of plantar fasciitis implies, plantar fasciitis has traditionally been considered an inflammatory process. However, recent findings suggest plantar fasciitis be a chronic degeneration causing marked thickening and fibrosis of the plantar fascia along with collagen necrosis, chondroid metaplasia, and calcification ${ }^{4}{ }^{10}$. Hence, it has been advocated that plantar fasciosism may be a more appropriate terminology compared to plantar fasciitis ${ }^{1,4)}$. While the diagnosis of plantar fasciitis is usually based on patient history, risk factors,

*Corresponding author. Weiqing Ge (E-mail: wge@ysu.edu)

(C)2017 The Society of Physical Therapy Science. Published by IPEC Inc.

This is an open-access article distributed under the terms of the Creative Commons Attribution Non-Commercial No Derivatives (by-nc-nd) License. (CC-BY-NC-ND 4.0: http://creativecommons.org/licenses/by-nc-nd/4.0/). 
and findings from physical examination ${ }^{1)}$, because the chronic degeneration healing mechanism is poorly understood, treatment of plantar fasciitis is often difficult ${ }^{4}$. Little convincing evidence is available to support various approaches for treating plantar fasciitis ${ }^{1}$. For example, mobilization of ankle and foot joint was recently found no more effective than stretching and ultrasound alone in treating plantar fasciitis ${ }^{11}$. For chronic recalcitrant plantar fasciitis that lasts more than six months after conservative treatment, surgery is recommended ${ }^{1}$. Additionally, recent evidence has demonstrated that dry needling can significantly reduce plantar heel pain ${ }^{12}$. However, the revised Clinical Practice Guidelines released by the Orthopaedic Section of the American Physical Therapy Association (APTA) titled "Heel Pain-Plantar Fasciitis: Revision 2014" states that "trigger point dry needling cannot be recommended for individuals with heel pain/plantar fasciitis"6).

Cupping therapy has recently gained the attention of the media and the public at the Rio Olympics, with extensive media coverage of the dark red circles left on Olympians' shoulders and backs, telltale signs of cupping therapy. Although cupping therapy has been performed in most cultures historically ${ }^{13}, 14$ ), this manual modality is mostly believed to be an ancient therapy primarily used in China and other Asian countries for thousands of years ${ }^{15}, 16$ ). This alternative therapy involves creating a vacuum inside a cup positioned over the surface of the skin, using the local negative pressure to promote blood flow ${ }^{17,18)}$. A few Systematic Reviews (SRs) of randomized clinical trials (RCTs) have been conducted to determine the effectiveness of cupping therapy in treating pain ${ }^{19)}$, hypertension ${ }^{20)}$, and stroke ${ }^{21)}$. The total number of RCTs meeting the inclusion criteria of the SRs were limited to determine the effectiveness ${ }^{19-21)}$. The quality of most of the RCTs were poor ${ }^{19-21)}$. Based on the currently available SRs, the effectiveness of cupping has been demonstrated only as a treatment for pain ${ }^{22)}$.

Cupping therapy may be a low-cost alternative to treat plantar fasciitis. To our knowledge, there is no research on the effectiveness of cupping therapy on pain and function for patients with plantar fasciitis. The purpose of this study was to determine the effects of dry cupping, a type of cupping therapy, on pain and function of patients with plantar fasciitis. The significance of this research project is that it may provide insight to physical therapy management of plantar fasciitis, particularly with regards to cupping therapy.

\section{SUBJECTS AND METHODS}

Twenty-nine subjects (age 15 to 59 years old, 20 females and 9 males) were recruited through a convenience sampling on the university campus using flyers posted on bulletin boards around campus and word of mouth. Data for body weight and height for each subject were not collected. The inclusion criteria included heel pain with a current or previous diagnosis of plantar fasciitis from a physician or with patient history, risk factors, and physical examination findings consistent with plantar fasciitis, and between 15 and 60 years of age. The exclusion criteria included contraindications to manual therapy or electrical stimulation, including tumors, recent fractures ( $<6$ months), rheumatoid arthritis, prolonged history of steroid use, severe vascular disease, open wounds, recent surgery to ankle joint or rear foot region ( $<6$ months), impaired sensation, pacemaker, and implants; inability to comply with treatment or the follow-up protocols; and currently undergoing other treatments for heel pain. Ethics approval for this study was sought and obtained from the Institutional Review Board at Youngstown State University. Written informed consent was obtained from each subject.

The research design was a randomized controlled trial. Subjects were randomly assigned to the dry cupping therapy (experimental) group ( $\mathrm{n}=14$, age 40.1 (SD 14.6) years old, 10 females and 4 males) or the electrical stimulation therapy (control) group ( $\mathrm{n}=15$, age 39.3 (SD 13.5) years old, 10 males and 5 females). There was no significant difference between the ages of the two groups $(\mathrm{p}=0.36)$.

The sample size was determined using $\mathrm{G}^{*}$ Power 3.1 (version 3.1.9.2) with the following parameters: effective size of 0.5 , alpha of 0.05 , and power of 0.80 . The total number of subjects was calculated to be 22 .

Treatments were provided to the subjects twice a week for 4 weeks. In the dry cupping therapy group, a plastic cupping bell (Kangzhu 6-Cup Biomagnetic Chinese Cupping Therapy Set, Model B1 × 6, Kangzhu, Beijing, China) was applied to the painful site for 10 minutes in each session. A manual hand pump was used to create the vacuum for suction. The intensity of the vacuum was based on subject tolerance. In the electrical stimulation therapy group, the subjects were provided with electrical stimulation therapy, a therapeutic modality routinely used by physical therapists for pain management, using a cabinet, multi-current stimulator (Dynatron Solaris 709, Dynatronics, Salt Lake City, UT, USA). The electrodes were placed around the painful site, and pre-modulated interferential current electrical stimulation was conducted for 10 minutes. The intensity of the current was increased to patient tolerance at the sensory level. The carrier frequencies were $4,000 \mathrm{~Hz}$ and 4,000-4,150 Hz. The beat frequency was 80-150 Hz.

Outcome measurements included the Visual Analogue Pain Scale (VAS) (at rest, first in the morning, and with activities), the Foot and Ankle Ability Measure (FAAM), the Lower Extremity Functional Scale (LEFS), as well as the pressure pain threshold. Pressure pain threshold was measured 3 times using a hand-held digital dynamometer (Lafayette Manual Muscle Tester Model 01163, Lafayette Instrument Company, Lafayette, IN, USA) at the most painful spot in the painful area. The subjects were instructed to report to the investigator when they started to feel pain or discomfort while the investigator gradually increased the force applied to the painful area through the dynamometer. A familiarization trial was conducted for pressure pain threshold for each subject. The pressure pain threshold was determined as the mean of the three trials. Outcomes were measured at baseline and at each session for VAS or every other session for all other outcome measurements.

Changes in VAS, FAAM, LEFS, and pain threshold are reported as means and 95\% confidence intervals (lower, upper 
$95 \%$ confidence interval). Student t-tests were used to determine whether there were statistically significant differences in the changes in the outcome measurements between the experimental and control groups. Significance was determined at $\alpha=0.05$.

\section{RESULTS}

The data indicated that both dry cupping therapy and electrical stimulation therapy could reduce pain and increase function significantly in the population tested. However, there was no significant difference between the dry cupping therapy and electrical stimulation groups in all the outcome measurements.

For the VAS, the mean changes in average score (at rest, first in the morning, and with activities) were $-29.8(-39.4$, $-20.1) \mathrm{mm}$ in the dry cupping therapy group compared to $-28.0(-36.7,-19.2) \mathrm{mm}$ in the electrical stimulation therapy group. There was no statistically significant difference between the two groups $(\mathrm{p}=0.39)$.

For the FAAM, the mean changes in score were $16.9(7.8,26.0) \%$ in the dry cupping therapy group compared to 12.9 $(8.2,17.6) \%$ in the electrical stimulation therapy group. There was no statistically significant difference between the two groups $(\mathrm{p}=0.27)$. The mean changes in patient perceived function were $12.3(7.6,17.0) \%$ in the dry cupping therapy group compared to $14.3(5.5,23.0) \%$ in the electrical stimulation therapy group. There was no statistically significant difference between the two groups $(\mathrm{p}=0.36)$.

For the LEFS, the mean changes in score were $19.6(8.6,30.7) \%$ in the dry cupping therapy group compared to $11.4(7.7$, $15.1) \%$ in the electrical stimulation therapy group. There was no statistically significant difference between the 2 groups $(\mathrm{p}=0.08)$.

For the pressure pain threshold, the mean changes in threshold were $4.6(0.0,9.1) \mathrm{lb}$ in the dry cupping therapy group compared to $1.7(-2.7,6.0) \mathrm{lb}$ in the electrical stimulation therapy group. There was no statistically significant difference between the 2 groups $(\mathrm{p}=0.19$ ). All the within-group changes were significant except for the pain threshold, as all the $95 \%$ CIs did not include 0 except for the pain threshold.

\section{DISCUSSION}

To our knowledge, this is the first study on the effectiveness of dry cupping therapy on the pain and function of patients with plantar fasciitis compared to electrical stimulation therapy. A recent Systematical Review on Traditional Chinese Medicine (TCM) identified that cupping therapy, along with acupuncture and acupressure, could be efficacious in treating pain and disability in patients with chronic neck pain or chronic low back pain ${ }^{23}$. In addition, cupping therapy has been used in treating various painful disorders $\left.{ }^{19}, 22\right)$, including low back pain ${ }^{24-27)}$, neck and shoulder pain ${ }^{28,29)}$, fibromyalgia ${ }^{30)}$, knee osteoarthritis $^{31,32)}$, and carpal tunnel syndrome ${ }^{33)}$. The results from this study add plantar fasciitis to the list. For all the outcome measurements, the cupping group did slightly better than the control group, although no statistically significant difference was found between the two groups. This research supports the integration of cupping therapy in treating plantar fasciitis in physical therapy practice.

We found that dry cupping therapy and electrical stimulation therapy had a similar level of effectiveness in decreasing pain and improving function in patients with plantar fasciitis. Interestingly, the therapeutic mechanisms of dry cupping therapy may be different from that of electrical stimulation therapy. Dry cupping therapy is believed to decrease pain by using the local negative pressure to promoting blood flow while electrical stimulation therapy by stimulating large-fiber sensory neurons and decreasing nociceptive inputs to central nervous system through the mechanisms of the gate control theory.

Cupping therapy can be performed using several different techniques ${ }^{18)}$. The two primary types are dry and wet cupping. Wet cupping, also called bleeding cupping, involves controlled bleeding ${ }^{18,25)}$, and hence may be prohibited by the current physical therapy practice laws in the United States. Cupping therapy can also be achieved using various subtypes of techniques, including retention, moving, shaking, quick, and balance cupping ${ }^{25}$. The vacuum required in cupping therapy for suction can be achieved using fire-heated air, manual hand pump, or electrical pump ${ }^{14,32,34)}$. In our study, dry cupping with retention technique and manual hand-pump was selected because it was easy to administer with relatively constant dosage and does not require expensive equipment. However, it should be noted that wet cupping is the most studied cupping technique in the literature, followed by dry (retained) cupping ${ }^{15,16)}$.

Institute of Medicine's Relieving Pain in America: a Blueprint for Transforming Prevention, Care, Education, and Research calls for a culture transformation to better prevent, assess, treat, and understand pain ${ }^{35)}$. The Blueprint advocates that the clinicians should increasingly aim at tailoring pain care to each person's experience, and self-management of pain by the patients should be promoted. Dry cupping is an ancient healing art that is easy to learn and suitable for self-management of pain. It does not require expensive equipment or a huge space to provide treatment for the patient.

The limitations of the research project include convenience sampling and the small sample size limited by our available resources. The convenience sampling significantly impacts the generalizability of the results. The subjects were mostly young volunteers, and the sample lacked diversity. In addition, double blindness was not possible to minimize subject and investigator biases. Future rigorous research with a larger sample size from physical therapy patients is necessary. 


\section{ACKNOWLEDGEMENT}

The authors thank DPT students Toby Gerez, Evan Wray, Joseph Osborn, and Adam Kaufman for their participation and contribution to the research project, and Vincent Ragozine, DPT, MS, for constructive discussions.

\section{REFERENCES}

1) Goff JD, Crawford R: Diagnosis and treatment of plantar fasciitis. Am Fam Physician, 2011, 84: 676-682. [Medline]

2) Healey K, Chen K: Plantar fasciitis: current diagnostic modalities and treatments. Clin Podiatr Med Surg, 2010, 27: 369-380. [Medline] [CrossRef]

3) Lim AT, How CH, Tan B: Management of plantar fasciitis in the outpatient setting. Singapore Med J, 2016, 57: 168-170, quiz 171. [Medline] [CrossRef]

4) Schwartz EN, Su J: Plantar fasciitis: a concise review. Perm J, 2014, 18: e105-e107. [Medline] [CrossRef]

5) McPoil TG, Martin RL, Cornwall MW, et al.: Heel pain — plantar fasciitis: clinical practice guildelines linked to the international classification of function, disability, and health from the orthopaedic section of the American Physical Therapy Association. J Orthop Sports Phys Ther, 2008, 38: A1-A18. [Medline] [CrossRef]

6) Martin RL, Davenport TE, Reischl SF, et al. American Physical Therapy Association: heel pain-plantar fasciitis: revision 2014. J Orthop Sports Phys Ther, 2014, 44: A1-A33. [Medline] [CrossRef]

7) Riddle DL, Pulisic M, Pidcoe P, et al.: Risk factors for plantar fasciitis: a matched case-control study. J Bone Joint Surg Am, 2003, 85-A: 872-877. [Medline] [CrossRef]

8) Tong KB, Furia J: Economic burden of plantar fasciitis treatment in the United States. Am J Orthop, 2010, 39: 227-231. [Medline]

9) Martinelli N, Bonifacini C, Romeo G: Current therapeutic approaches for plantar fasciitis. Orthop Res Rev, 2014, 6: 33-40. [CrossRef]

10) Thompson JV, Saini SS, Reb CW, et al.: Diagnosis and management of plantar fasciitis. J Am Osteopath Assoc, 2014, 114: 900-906. [Medline] [CrossRef]

11) Shashua A, Flechter S, Avidan L, et al.: The effect of additional ankle and midfoot mobilizations on plantar fasciitis: a randomized controlled trial. J Orthop Sports Phys Ther, 2015, 45: 265-272. [Medline] [CrossRef]

12) Cotchett MP, Munteanu SE, Landorf KB: Effectiveness of trigger point dry needling for plantar heel pain: a randomized controlled trial. Phys Ther, 2014, 94: 1083-1094. [Medline] [CrossRef]

13) Chen B, Li MY, Liu PD, et al.: Alternative medicine: an update on cupping therapy. QJM, 2015, 108: 523-525. [Medline] [CrossRef]

14) Rozenfeld E, Kalichman L: New is the well-forgotten old: the use of dry cupping in musculoskeletal medicine. J Bodyw Mov Ther, 2016, 20: 173-178. [Medline] [CrossRef]

15) Cao H, Han M, Li X, et al.: Clinical research evidence of cupping therapy in China: a systematic literature review. BMC Complement Altern Med, 2010, 10: 70. [Medline] [CrossRef]

16) Cao H, Li X, Liu J: An updated review of the efficacy of cupping therapy. PLoS One, 2012, 7: e31793. [Medline] [CrossRef]

17) Tham LM, Lee HP, Lu C: Cupping: from a biomechanical perspective. J Biomech, 2006, 39: 2183-2193. [Medline] [CrossRef]

18) Yoo SS, Tausk F: Cupping: East meets West. Int J Dermatol, 2004, 43: 664-665. [Medline] [CrossRef]

19) Kim JI, Lee MS, Lee DH, et al.: Cupping for treating pain: a systematic review. Evid Based Complement Alternat Med, 2011, 2011: 467014. [Medline] [CrossRef]

20) Lee MS, Choi TY, Shin BC, et al.: Cupping for hypertension: a systematic review. Clin Exp Hypertens, 2010, 32: 423-425. [Medline] [CrossRef]

21) Lee MS, Choi TY, Shin BC, et al.: Cupping for stroke rehabilitation: a systematic review. J Neurol Sci, 2010, 294: 70-73. [Medline] [CrossRef]

22) Lee MS, Kim JI, Ernst E: Is cupping an effective treatment? An overview of systematic reviews. J Acupunct Meridian Stud, 2011, 4: 1-4. [Medline] [CrossRef]

23) Yuan QL, Guo TM, Liu L, et al.: Traditional Chinese medicine for neck pain and low back pain: a systematic review and meta-analysis. PLoS One, 2015, 10: e0117146. [Medline] [CrossRef]

24) AlBedah A, Khalil M, Elolemy A, et al.: The use of wet cupping for persistent nonspecific low back pain: randomized controlled clinical trial. J Altern Complement Med, 2015, 21: 504-508. [Medline] [CrossRef]

25) Huang CY, Choong MY, Li TS: Effectiveness of cupping therapy for low back pain: a systematic review. Acupunct Med, 2013, 31: 336-337. [Medline] [CrossRef]

26) Kim JI, Kim TH, Lee MS, et al.: Evaluation of wet-cupping therapy for persistent non-specific low back pain: a randomised, waiting-list controlled, open-label, parallel-group pilot trial. Trials, 2011, 12: 146. [Medline] [CrossRef]

27) Lauche R, Cramer H, Hohmann C, et al.: The effect of traditional cupping on pain and mechanical thresholds in patients with chronic nonspecific neck pain: a randomised controlled pilot study. Evid Based Complement Alternat Med, 2012, 2012: 429718. [Medline] [CrossRef]

28) Arslan M, Gökgöz N, Dane Ş: The effect of traditional wet cupping on shoulder pain and neck pain: a pilot study. Complement Ther Clin Pract, 2016, 23: 30-33. [Medline] [CrossRef]

29) Chi LM, Lin LM, Chen CL, et al.: The effectiveness of cupping therapy on relieving chronic neck and shoulder pain: a randomized controlled trial. Evid Based Complement Alternat Med, 2016, 2016: 7358918. [Medline] [CrossRef]

30) Lauche R, Spitzer J, Schwahn B, et al.: Efficacy of cupping therapy in patients with the fibromyalgia syndrome-a randomised placebo controlled trial. Sci Rep, 2016, 6: 37316. [Medline] [CrossRef]

31) Khan AA, Jahangir U, Urooj S: Management of knee osteoarthritis with cupping therapy. J Adv Pharm Technol Res, 2013, 4: 217-223. [Medline] [CrossRef]

32) Teut M, Kaiser S, Ortiz M, et al.: Pulsatile dry cupping in patients with osteoarthritis of the knee — a randomized controlled exploratory trial. BMC Complement Altern Med, 2012, 12: 184. [Medline] [CrossRef]

33) Michalsen A, Bock S, Lüdtke R, et al.: Effects of traditional cupping therapy in patients with carpal tunnel syndrome: a randomized controlled trial. J Pain, 2009, 10: 601-608. [Medline] [CrossRef]

34) Huang S, Cao Y: Cupping therapy. J Chin Med. 2006, 82: 52-57.

35) Institute of Medicine (U.S.) Committee on Advancing Pain Research: Relieving pain in America: a blueprint for transforming prevention, care, education, and research. Washington DC: National Academies Press, 2011. http://site.ebrary.com/id/10520732. 